\title{
ON A CLASS OF CLOSE-TO-CONVEX FUNCTIONS
}

\author{
H. SILVERMAN
}

ABSTRACT. We look at functions $f(z)$ for which there correspond functions $\phi(z)$ convex of order $\alpha$ such that $\operatorname{Re}\left\{f^{\prime}(z) / \phi^{\prime}(z)\right\} \geqq \beta$. We examine the influence of the second coefficient of $\phi(z)$ on this class. In particular, distortion, covering, and radius of convexity theorems are proved.

1. Introduction. Let $S$ be the class of normalized univalent functions analytic in the unit disk $E(|z|<1)$. Let $K_{p}(\alpha)$ denote the subclass of $S$ consisting of functions of the form $\phi(z)=z+b_{2} z^{2}+\cdots$, where

$$
\operatorname{Re}\left\{z \phi^{\prime \prime}(z) / \phi^{\prime}(z)+1\right\} \geqq \alpha, \quad z \in E,\left|b_{2}\right|=p,
$$

and $0 \leqq \alpha \leqq 1$. This class is called convex of order $\alpha$. It is known that $0 \leqq p \leqq 1$. In addition, $\phi(z) \in K_{p}(1)$ if and only if $\phi(z)=z$ and $\phi(z) \in K_{1}(\alpha)$ if and only if $\phi(z)=z /(1-x z),|x|=1$.

We say that an analytic function $f(z)=z+a_{2} z^{2}+\cdots$ is in the class $C_{p}(\alpha, \beta)$ if there exists a function $\phi(z) \in K_{p}(\alpha)$ such that

$$
\operatorname{Re}\left\{f^{\prime}(z) / \phi^{\prime}(z)\right\} \geqq \beta, \quad \beta \geqq 0 .
$$

It is easy to verify that for $\alpha \leqq \alpha_{0}$ and $\beta \leqq \beta_{0}$ we have

$$
C_{p}\left(\alpha_{0}, \beta\right) \subset C_{p}(\alpha, \beta) \text { and } C_{p}\left(\alpha, \beta_{0}\right) \subset C_{p}(\alpha, \beta) \text {. }
$$

Kaplan [5] proved that $C_{p}(0,0)$, the class of close-to-convex functions, is univalent. Hence $C_{p}(\alpha, \beta)$ is a subclass of $S$.

By specializing $\alpha$ and $\beta$ we obtain some important classes. If $f(z)$ is in $C_{p}(0,0)$, then $f(z)$ is close-to-convex; $C_{p}(1, \beta)$, then $\operatorname{Re} f^{\prime}(z) \geqq \beta$; $C_{p}(\alpha, 1)$, then $f(z)$ is convex of order $\alpha ; C_{p}(1,1)$, then $f(z)=z$.

In this note we prove distortion, covering, and radius of convexity theorems for the class $C_{p}(\alpha, \beta)$. By specializing $p$, some of our results will coincide with those of Libera [6]. We also look at a corresponding subclass of the close-to-star functions, $S_{p}(\alpha, \beta)$. Some of our results for this class will generalize those of Al-Amiri [1] who investigated the class $S_{n}(\alpha, 0)$.

Received by the editors November 8, 1971 and, in revised form, February 17, 1972. AMS 1969 subject classifications. Primary 3032; Secondary 3036.

Key words and phrases. Univalent, starlike, convex, close-to-convex, radius of convexity. 
In the sequel, we will assume that $f(z)$ is in $C_{p}(\alpha, \beta)$ with $\phi(z)$ its associated function in $K_{p}(\alpha)$.

2. Distortion theorems for $C_{p}(\alpha, \beta)$. We begin by proving an existence theorem for functions in this class.

TheOREM 1. Let $\alpha \in[0,1], \beta \in[0,1]$, and $p \in[0,1-\alpha]$. Then there exists a function $f(z) \in C_{p}(\alpha, \beta)$. This result is sharp in that $\alpha+p \leqq 1$ for any $\alpha$.

In proving the theorem we will make use of the following

Lemma. Let $Q(z)$ be analytic for $z \in E$ with $Q(0)=1$. Then $\operatorname{Re} Q(z) \geqq \beta$ if and only if

$$
Q(z)=\frac{1+(1-2 \beta) g(z)}{1-g(z)},
$$

where $g(z)$ is analytic, $g(0)=0$, and $|g(z)|<1$ for $z \in E$.

Proof of Lemma. The result is well known for $\alpha=0$. In the general case, let $Q(z)=(1-\alpha) P(z)+\alpha$, where $P(z)$ satisfies the conditions of the Lemma with $\alpha=0$.

Proof Of TheOREM 1. The inequality $\alpha+p \leqq 1$ is proved in [1, p. 104]. Let

$$
f(z)=\int_{0}^{z}\left(\frac{1}{1-t^{2}}\right)^{1-\alpha}\left(\frac{1+t}{1-t}\right)^{p}\left(\frac{1+(1-2 \beta) t}{1-t}\right) d t
$$

and

Since

$$
\phi(z)=\int_{0}^{z}\left(\frac{1}{1-t^{2}}\right)^{1-\alpha}\left(\frac{1+t}{1-t}\right)^{p} d t
$$

$$
\frac{f^{\prime}(z)}{\phi^{\prime}(z)}=\frac{1+(1-2 \beta) z}{1-z}
$$

has real part $\geqq \beta$, it suffices to show that $\phi(z) \in K_{p}(\alpha)$.

We have

$$
1+z \frac{\phi^{\prime \prime}(z)}{\phi^{\prime}(z)}=\frac{1+2 p z+(1-2 \alpha) z^{2}}{1-z^{2}}=\frac{1+(1-2 \alpha) g(z)}{1-g(z)} .
$$

Solving for $g(z)$, we obtain

$$
g(z)=z\left(\frac{z+p /(1-\alpha)}{1+(p /(1-\alpha)) z}\right)=z h(z) .
$$

Since $\alpha+p \leqq 1, h(z)$ maps $E \rightarrow E$, and $|g(z)| \leqq|z|<1$ for $z \in E$. Since $g(z)$ satisfies the conditions of the Lemma, our proof is complete. 
THEOREM 2. Let $f(z) \in C_{p}(\alpha, \beta)$. Then

$$
\begin{aligned}
& \left|f^{\prime}(z)\right| \leqq\left(\frac{1}{1-r^{2}}\right)^{1-\alpha}\left(\frac{1+r}{1-r}\right)^{p}\left(\frac{1+(1-2 \beta) r}{1-r}\right), \\
& \left|f^{\prime}(z)\right| \geqq \frac{1}{\left(1+(2 p /(1-\alpha)) r+r^{2}\right)^{1-\alpha}}\left(\frac{1-(1-2 \beta) r}{1+r}\right),
\end{aligned}
$$

where the first expression on the right-hand side of (2) is taken to be 1 for $\alpha=1$.

Equality holds in (1) for the function

$$
f_{1}(z)=\int_{0}^{z}\left(\frac{1}{1-t^{2}}\right)^{1-x}\left(\frac{1+t}{1-t}\right)^{n}\left(\frac{1+(1-2 \beta) t}{1-t}\right) d t
$$

and equality holds in (2) for the function

$$
f_{2}(z)=\int_{0}^{z} \frac{1}{\left(1+(2 p /(1-\alpha)) t+t^{2}\right)^{1-\alpha}}\left(\frac{1-(1-2 \beta) t}{1+t}\right) d t .
$$

Proof. From the Lemma we obtain

$$
\frac{f^{\prime}(z)}{\phi^{\prime}(z)}=\frac{1+(1-2 \beta) g(z)}{1-g(z)}
$$

where $g(0)=0$ and $|g(z)|<1$ for $z \in E$.

Since $g(z)$ satisfies the conditions of Schwarz's lemma, (3) yields

$$
\frac{1-(1-2 \beta) r}{1+r} \leqq\left|\frac{f^{\prime}(z)}{\phi^{\prime}(z)}\right| \leqq \frac{1+(1-2 \beta) r}{1-r} .
$$

In $[\mathbf{1}, \mathrm{p} .105]$ it is proved that

$$
\frac{1}{\left(1+(2 p /(1-\alpha)) r+r^{2}\right)^{1-\alpha}} \leqq\left|p^{\prime}(z)\right| \leqq\left(\frac{1}{1-r^{2}}\right)^{1-\alpha}\left(\frac{1+r}{1-r}\right)^{n}
$$

Combining (4) and (5), the result follows. In the proof of Theorem 1 it was shown that $f_{1}(z) \in C_{p}(\alpha, \beta)$. The proof that $f_{2}(z) \in C_{p}(\alpha, \beta)$ is similar, with

$$
\phi_{2}(z)=\int_{0}^{z} \frac{1}{\left(1+(2 p /(1-\alpha)) t+t^{2}\right)^{1-\alpha}} d t .
$$

Remark. For $p=1-\alpha$, (1) reduces to a result of Libera and (2) improves on a result of Libera $[6$, p. 152]. In his paper, it is claimed that 
the function

$$
f(z)=\int_{0}^{z} \frac{1-t}{(1+t)^{2(1-\alpha)}(1+t(1-2 \beta))} d t
$$

is in $C_{1-\alpha}(\alpha, \beta)$ for every $\alpha$ and $\beta$. That this is not the case can be seen by letting $\alpha=1$ and $\beta={ }_{2}^{1}$. Then $f(z)=z-z^{2} / 2$ and $\phi(z)=z$. But

$$
\operatorname{Re}\left\{f^{\prime}(z) / \phi^{\prime}(z)\right\}=\operatorname{Re}\{1-z\}
$$

which is less than $\underset{2}{1}$ for $\underset{2}{1}<z<1$, and $f(z)=z-z^{2} / 2 \notin C_{1-\alpha}\left(1, \frac{1}{2}\right)$.

TheOREM 3. Let $f(z) \in C_{p}(\alpha, \beta)$. Then

$$
\begin{aligned}
\int_{0}^{r} \frac{1}{\left(1+(2 p /(1-\alpha)) t+t^{2}\right)^{1-\alpha}}\left(\frac{1-(1-2 \beta) t}{1+t}\right) d t \leqq|f(z)| \\
\leqq \int_{0}^{r}\left(\frac{1}{1-t^{2}}\right)^{1-\alpha}\left(\frac{1+t}{1-t}\right)^{p}\left(\frac{1-(1-2 \beta) t}{1+t}\right) d t .
\end{aligned}
$$

Equality holds on the right-hand side for $f_{1}(z)$ in Theorem 2 and on the lefthand side for $f_{2}(z)$ in Theorem 2.

Proof. Integrating along the straight line segment from the origin to $z=r e^{i \theta}$ and applying Theorem 2 we obtain

$$
|f(z)| \leqq \int_{0}^{r}\left|f^{\prime}\left(t e^{i \theta}\right)\right| d t \leqq \int_{0}^{r}\left(\frac{1}{1-t^{2}}\right)^{1-\alpha}\left(\frac{1+t}{1-t}\right)^{p}\left(\frac{1-(1-2 \beta) t}{1+t}\right) d t
$$

which proves the right-hand inequality. To prove the left-hand inequality, for every $r$ we choose $z_{0},\left|z_{0}\right|=r$, such that

$$
\left|f\left(z_{0}\right)\right|=\min _{|z|=r}|f(z)| .
$$

If $L\left(z_{0}\right)$ is the pre-image of the segment $\left\{0, f\left(z_{0}\right)\right\}$, then

$$
\begin{aligned}
|f(z)| & \geqq\left|f\left(z_{0}\right)\right| \geqq \int_{L\left(z_{0}\right)}\left|f^{\prime}(z)\right||d z| \\
& \geqq \int_{0}^{r} \frac{1}{\left(1+(2 p /(1-\alpha)) t+t^{2}\right)^{1-\alpha}}\left(\frac{1-(1-2 \beta) t}{1+t}\right) d t
\end{aligned}
$$

and this completes the proof.

For $p=1-\alpha$, the right-hand inequality reduces to a result of Libera and the left-hand inequality sharpens a result of Libera [6, p. 152].

3. Covering theorems for $C_{p}(\alpha, \beta)$. We first prove a coefficient theorem for the class. 
THEOREM 4. Let $f(z) \in C_{p}(\alpha, \beta)$, with $f(z)=z+\sum_{n=2}^{\infty} a_{n} z^{n}$. Then $\left|a_{2}\right| \leqq 1+p-\beta$, with extremal function

$$
f(z)=\int_{0}^{z}\left(\frac{1}{1-t^{2}}\right)^{1-\alpha}\left(\frac{1+t}{1-t}\right)^{p}\left(\frac{1+(1-2 \beta) t}{1-t}\right) d t .
$$

Proof. Let $\phi(z)=z+\sum_{n=2}^{\infty} b_{n} z^{n}$. Then

$$
g(z)=\frac{f^{\prime}(z) / \varphi^{\prime}(z)-\beta}{1-\beta}=1+\frac{2\left(a_{2}-b_{2}\right)}{1-\beta}+\sum_{n=2}^{\infty} c_{n} z^{n}
$$

has positive real part in $E$. Hence [3, p. 15], $2\left|a_{2}-b_{2}\right| /(1-\beta) \leqq 2$, or $\left|a_{2}\right| \leqq 1+\left|b_{2}\right|-\beta=1+p-\beta$.

THEOREM 5. Let $f(z) \in C_{p}(\alpha, \beta)$, with $f(z)=z+\sum_{n=2}^{\infty} a_{n} z^{n}$.

If $f(z) \neq k$ for $z \in E$, then $|k| \geqq 1 /(3+p-\beta)$.

Proof. If $f(z)$ does not assume the value $k$, then

$$
\frac{k f(z)}{k-f(z)}=z+\left(a_{2}+1 / k\right) z^{2}+\sum_{n=3}^{\infty} c_{n} z^{n}
$$

is in the class $S$. Hence,

$$
\left|a_{2}+1 / k\right| \leqq 2
$$

Applying the triangle inequality and Theorem 4 to (6) we obtain Theorem 5 .

Since $p \leqq 1-\alpha$, we also obtain the following result of Libera [6, p. 155] as a

COROLlaRY. $|k| \geqq 1 /(4-\alpha-\beta)$.

4. A radius of convexity theorem for $C_{p}(\alpha, \beta)$.

THEOREM 6. Let $f(z) \in K_{p}(\alpha, \beta)$. Then $f(z)$ maps the disk $|z|<R$ onto a convex domain, where $R$ is the least positive root of the equation $a(r, p, \alpha, \beta)=0$, where

$$
\begin{aligned}
a(r, p, \alpha, \beta)= & (1-\alpha)(1-2 \alpha)(1-2 \beta) r^{4} \\
& -2[(1-\alpha)(1-\beta)+\alpha p(1-2 \beta) \\
& -\beta(1-\alpha)(1-2 \alpha)] r^{3} \\
& -2[(1-\alpha)(1-\alpha-\beta-2 p \beta)+2 p] r^{2} \\
& -2[1-\alpha(1+p)] r+(1-\alpha) .
\end{aligned}
$$

Proof. Let $f^{\prime}(z) / \phi^{\prime}(z)=Q(z)$, where $\operatorname{Re} Q(z) \geqq \beta$. Since

$$
z \frac{f^{\prime \prime}(z)}{f^{\prime}(z)}=z \frac{\phi^{\prime \prime}(z)}{\phi^{\prime}(z)}+z \frac{Q^{\prime}(z)}{Q(z)},
$$


the radius of convexity of $f(z)$ is at least equal to the smallest positive root of

$$
1+\min \operatorname{Re}\left\{z \phi^{\prime \prime}(z) / \phi^{\prime}(z)\right\}+\min \operatorname{Re}\left\{z Q^{\prime}(z) / Q(z)\right\}=0 .
$$

In $[1$, p. 105], it is shown that

$$
\operatorname{Re}\left\{z \frac{\phi^{\prime \prime}(z)}{\phi^{\prime}(z)}\right\} \geqq-\frac{2 r(1-\alpha)[r(1-\alpha)+p]}{(1-\alpha)\left(1+r^{2}\right)+2 p r} .
$$

Now let $Q(z)=(1-\beta) P(z)+\beta$, where $P(z)$ is analytic, $P(0)=1$, and $\operatorname{Re} P(z)>0$ in $E$. Then

$$
z \frac{Q^{\prime}(z)}{Q(z)}=\frac{(1-\beta) P^{\prime}(z)}{(1-\beta) P(z)+\beta}=\frac{P^{\prime}(z)}{P(z)+\beta /(1-\beta)} .
$$

Using a lemma of Libera [6, p. 150] we obtain

$$
\left|z \frac{Q^{\prime}(z)}{Q(z)}\right| \leqq \frac{2 r}{(1-r)[1+r+(\beta /(1-\beta))(1-r)]} .
$$

Substituting (8) and (9) into (7) yields

$$
\begin{aligned}
\operatorname{Re}\left\{1+z \frac{f^{\prime \prime}(z)}{f^{\prime}(z)}\right\} \geqq & 1-\frac{2 r(1-\alpha)[r(1-\alpha)+p]}{(1-\alpha)\left(1+r^{2}\right)+2 p r} \\
& -\frac{2 r}{(1-r)[1+r+(\beta /(1-\beta))(1-r)]} .
\end{aligned}
$$

Simplifying the right-hand side of (10) we obtain

$$
\frac{a(r, p, \alpha, \beta)}{\left[\left(1-\alpha_{)}\right)\left(1+r^{2}\right)+2 p r\right][(1-r)(1+r+(\beta /(1-\beta))(1-r)]},
$$

and this completes the proof.

REMARK 1. The Koebe function is in $C_{1}(0,0)$, and the least positive root of

$$
a(r, 1,0,0)=r^{4}-2 r^{3}-6 r^{2}-2 r+1
$$

is $2-\sqrt{3}$, the radius of convexity for the class $S$.

REMARK 2. If $p=1-\alpha$,

$$
\begin{aligned}
& a(r, 1-\alpha, \alpha, \beta) \\
& =(r+1)(1-\alpha)\left[(1-2 \alpha)(1-2 \beta) r^{3}\right. \\
& \left.-(3-6 \beta+4 \alpha \beta) r^{2}+(2 \alpha-3) r+1\right] .
\end{aligned}
$$

This reduces to a result of Libera [6, p. 151]. 
5. The class $S_{p}(\alpha, \beta)$. Let $S_{p}^{*}(\alpha)$ denote the class of functions analytic in $E$ and of the form $s(z)=z+a_{2} z^{2}+\cdots$, where $s(z)$ is starlike of order $\alpha$ and $\left|a_{2}\right|=2 p$.

Let $S_{p}(\alpha, \beta)$ denote the class of functions analytic in $E$ and of the form $g(z)=z+b_{2} z^{2}+\cdots$ such that $\operatorname{Re}\{g(z) / s(z)\} \geqq \beta$ for $z \in E$ and for some $s(z) \in S_{p}^{*}(\alpha)$.

The class $S_{p}(0,0)$, defined by Reade [7], is called close-to-star. It is known that members of this class need not be univalent. However, there is an important connection between the classes $S_{p}(\alpha, \beta)$ and $C_{p}(\alpha, \beta)$ which we state as

THEOREM 7. The following relationships hold:

$$
\begin{aligned}
& f(z) \in C_{p}(\alpha, \beta) \quad \text { if and only if } \quad z f^{\prime}(z) \in S_{p}(\alpha, \beta), \\
& f(z) \in S_{p}(\alpha, \beta) \quad \text { if and only if } \int_{0}^{z} \frac{f(t)}{t} d t \in C_{p}(\alpha, \beta) .
\end{aligned}
$$

Proof. It is well known that $\phi(z)$ is convex of order $\alpha$ if and only if $z \phi^{\prime}(z)$ is starlike of order $\alpha$. Hence, $\phi(z)=z+a_{2} z^{2}+\cdots$ is in $K_{p}(\alpha)$ if and only if $z \phi^{\prime}(z)=z+2 a_{2} z^{2}+\cdots$ is in $S_{p}^{*}(\alpha)$. Since

$$
\operatorname{Re}\left\{f^{\prime}(z) / \phi^{\prime}(z)\right\}=\operatorname{Re}\left\{z f^{\prime}(z) / z \phi^{\prime}(z)\right\},
$$

we obtain (11). The proof of (12) is similar and will be omitted.

TheOREM 8. Let $g(z) \in S_{\jmath}(\alpha, \beta)$. Then

$$
\begin{aligned}
\frac{r}{\left(1+(2 p /(1-\alpha)) r+r^{2}\right)^{1-\alpha}} & \left(\frac{1-(1-2 \beta) r}{1+r}\right) \leqq|g(z)| \\
& \leqq \frac{r}{\left(1-r^{2}\right)^{1-\alpha}}\left(\frac{1+r}{1-r}\right)^{n}\left(\frac{1+(1-2 \beta) r}{1-r}\right) .
\end{aligned}
$$

Proof. The result follows on combining Theorem 2 with Theorem 7.

Corollary. Let $g(z)$ be analytic in $E$ with $\operatorname{Re}\{g(z) / z\}>?$. Then

$$
r /(1+r) \leqq|g(z)| \leqq r /(1-r)
$$

Proof. We have $\operatorname{Re}\{g(z) / z\}>\frac{1}{2}$ if and only if $g(z) \in S_{0}\left(1, \frac{1}{2}\right)$.

Schild [8, p. 752] proved (13) for the class of functions starlike of order $\frac{1}{2}$, a subclass of $S_{0}\left(1, \frac{1}{2}\right)[4$, p. 472$]$.

For a functional analysis proof of the corollary, see [2, p. 94].

Once again making use of Theorem 7 , we see that $g(z) \in S_{p}(\alpha, \beta)$ if and only if

$$
z \frac{g^{\prime}(z)}{g(z)}=1+z \frac{f^{\prime \prime}(z)}{f^{\prime}(z)}
$$


for some $f(z) \in C_{p}(\alpha, \beta)$. Hence a radius of convexity theorem in $C_{p}(\alpha, \beta)$ will correspond to a radius of starlikeness theorem in $S_{p}(\alpha, \beta)$. From Theorem 6 we now obtain

THEOREM 9. Let $g(z) \in S_{p}(\alpha, \beta)$. Then $g(z)$ maps the disk $|z|<R$ onto a starlike domain, where $R$ is the least positive root of $a(r, p, \alpha, \beta)=0$, defined in Theorem 6.

For $\beta=0$, this reduces to a result of Al-Amiri [1, p. 108].

\section{BIBLIOGRAPHY}

1. H. S. Al-Amiri, On p-close-to-star functions of order $\alpha$, Proc. Amer. Math. Soc. 29 (1971), 103-108.

2. L. Brickman, T. H. MacGregor and D. R. Wilken, Convex hulls of some classical families of univalent functions, Trans. Amer. Math. Soc. 156 (1971), 91-107. MR 43 \#494.

3. W. K. Hayman, Multivalent functions, Cambridge Tracts in Math. and Math. Phys., no. 48, Cambridge Univ. Press, Cambridge, 1958. MR 21 \#7302.

4. I. S. Jack, Functions starlike and convex of order $\alpha$, J. London Math. Soc. (2) 3 (1971), 469-474.

5. W. Kaplan, Close-to-convex schlicht functions, Michigan Math. J. 1 (1952), 169-185. MR 14, 966.

6. R. J. Libera, Some radius of convexity problems, Duke Math. J. 31 (1964), 143158. MR 28 \#4099.

7. M. O. Reade, On close-to-convex univalent functions, Michigan Math. J. 3 (1955), 59-62. MR 17, 25.

8. A. Schild, On a class of univalent, star-shaped mappings, Proc. Amer. Math. Soc. 9 (1958), 751-757. MR 20 \#2452.

Department of Mathematics, Clark University, Worcester, Massachusetts 01610 